PROCEEDINGS OF THE

AMERICAN MATHEMATICAL SOCIETY

Volume 134, Number 5, Pages 1319-1329

S 0002-9939(05)08278-X

Article electronically published on October 4, 2005

\title{
SOBOLEV SPACES AND THE CAYLEY TRANSFORM
}

\author{
FRANCESCA ASTENGO AND BIANCA DI BLASIO
}

(Communicated by Andreas Seeger)

\begin{abstract}
The generalised Cayley transform $\mathcal{C}$ from an Iwasawa $N$-group into the corresponding real unit sphere $\mathbb{S}$ induces isomorphisms between suitable Sobolev spaces $\mathcal{H}^{\alpha}(\mathbb{S})$ and $\mathcal{H}^{\alpha}(N)$. We study the differential of $\mathcal{C}$, and we obtain a criterion for a function to be in $\mathcal{H}^{\alpha}(\mathbb{S})$.
\end{abstract}

\section{INTRODUCTION}

The classical stereographic projection is a conformal bijection from $\mathbb{R}^{n} \cup\{\infty\}$ to $\mathbb{S}$, the unit sphere in $\mathbb{R}^{n+1}$, where $n \geq 2$. In $[5]$ the authors generalised the stereographic projection to the case where $\mathbb{R}^{n}$ is replaced by a nilpotent Lie group $N$ of Heisenberg type and the sphere is the Euclidean sphere in a suitable one-dimensional extension of the Lie algebra of $N$. They call this map (generalised) Cayley transform.

The class of Heisenberg type groups was introduced by Kaplan in 10, and it includes all Iwasawa $N$-groups associated to real rank-one simple Lie groups. In [4] it was proved that the Iwasawa $N$-groups are characterised among all Heisenberg type groups by a Lie-algebraic condition, the so-called $J^{2}$-condition. In this case, the Cayley transform $\mathcal{C}: N \longrightarrow \mathbb{S}$ is a conformal map with respect to suitable CarnotCaratheodory structures on $N$ and on the sphere [1, 2]. As Carnot-Caratheodory manifolds, $N$ and $\mathbb{S}$ have privileged subspaces $T_{n}^{(1)}(N)$ and $T_{\mathcal{C}(n)}^{(1)}(\mathbb{S})$ of the tangent spaces at any points $n$ in $N$ and $p=\mathcal{C}(n)$ in $\mathbb{S}$. From the fact that $\mathcal{C}$ is conformal it follows that its differential $\mathcal{C}_{*}$ maps $T_{n}^{(1)}(N)$ into $T_{\mathcal{C}(n)}^{(1)}(\mathbb{S})$ and, restricted to $T_{n}^{(1)}(N)$, is a multiple of an isometry [1. For more information on conformal and quasiconformal mappings on boundaries of symmetric spaces see [11, 12, 13].

In Section 3 of this paper we study the Cayley transform in the case where $N$ is not necessarily an Iwasawa group: we compute the differential $\mathcal{C}_{*}$ and we give an elementary proof of the fact that the $J^{2}$-condition holds if and only if the restriction of $\mathcal{C}_{*}$ to $T_{n}^{(1)}(N)$ is a multiple of an isometry.

In Section 4 we consider Sobolev spaces on $\mathbb{S}$ and an Iwasawa $N$-group defined in terms of powers of suitable conformal sublaplacians. In 1 it was proved that the Cayley transform induces isomorphisms from Sobolev spaces $\mathcal{H}^{\alpha}(\mathbb{S})$ to $\mathcal{H}^{\alpha}(N)$. In

Received by the editors November 18, 2004.

2000 Mathematics Subject Classification. Primary 43A80; Secondary 43A85 43A15.

Key words and phrases. Heisenberg type groups, Sobolev spaces.

The authors thank the School of Mathematics of the University of the New South Wales and the Italian G.N.A.M.P.A. for their support. 
Section 4 we find an explicit formula for the conformal sublaplacian on the sphere, and we use this result to obtain a useful criterion for a function to be in a Sobolev space on the sphere. The peculiarity of this criterion is that it involves vector fields on the sphere which are not rotation-invariant, nor divergence-free.

We use the "variable constant convention", according to which constants are denoted by $C, C^{\prime}$, etc., and these are not necessarily equal at different occurrences. All "constants" are positive.

It is a pleasure to thank Michael Cowling for many helpful conversation on the subject of this paper.

\section{NOTATION AND PRELIMINARIES}

Let $\mathfrak{n}$ be a two-step real nilpotent Lie algebra, with an inner product $\langle\cdot, \cdot\rangle$ such that $\mathfrak{n}$ may be written as an orthogonal sum $\mathfrak{n}=\mathfrak{v} \oplus \mathfrak{z}$, where $\mathfrak{z}$ is the center of $\mathfrak{n}$. For each $Z$ in $\mathfrak{z}$, define the map $J_{Z}: \mathfrak{v} \longrightarrow \mathfrak{v}$ by the formula

$$
\left\langle J_{Z} X, Y\right\rangle=\langle[X, Y], Z\rangle \quad \forall X, Y \in \mathfrak{v} .
$$

Following Kaplan [10, we say that the Lie algebra $\mathfrak{n}$ is $H$-type if

$$
J_{Z}^{2}=-|Z|^{2} I_{\mathfrak{v}} \quad \forall Z \in \mathfrak{z},
$$

where $I_{\mathfrak{v}}$ is the identity operator on $\mathfrak{v}$. A connected, simply-connected Lie group $N$ whose Lie algebra is an $H$-type algebra is said to be a Heisenberg type group or $H$-type group for short. The Iwasawa $N$-groups associated to all real rank-one simple groups are $H$-type. We denote the dimension of the center $\mathfrak{z}$ by $d_{\mathfrak{z}}$ and the dimension of $\mathfrak{v}$ by $d_{\mathfrak{v}}$. Note that from property (2.1) it follows that $\mathfrak{z}=[\mathfrak{v}, \mathfrak{v}]$, and moreover, if $\mathfrak{z}$ is nontrivial, the dimension $d_{\mathfrak{v}}$ of $\mathfrak{v}$ is even.

We shall use the following properties of the map $J$. These properties are proved in [4, Section 1]: for every $X, X^{\prime}$ in $\mathfrak{v}$ and $Z, Z^{\prime}$ in $\mathfrak{z}$

$$
\begin{aligned}
J_{Z} J_{Z^{\prime}}+J_{Z^{\prime}} J_{Z} & =-2\left\langle Z, Z^{\prime}\right\rangle I_{\mathfrak{v}}, \\
\left\langle J_{Z} X, J_{Z^{\prime}} X^{\prime}\right\rangle+\left\langle J_{Z^{\prime}} X, J_{Z} X^{\prime}\right\rangle & =2\left\langle Z, Z^{\prime}\right\rangle\left\langle X, X^{\prime}\right\rangle, \\
J_{\left[X, X^{\prime}\right]} X & =|X|^{2} P_{J_{\mathfrak{z}} X} X^{\prime}, \\
{\left[X, J_{Z} X\right] } & =|X|^{2} Z,
\end{aligned}
$$

where $P_{J_{3} X} X^{\prime}$ is the projection of the vector $X^{\prime}$ on the space

$$
J_{\mathfrak{z}} X=\left\{J_{Z} X: Z \in \mathfrak{z}\right\} .
$$

Since $N$ is a simply-connected nilpotent Lie group, the exponential mapping is bijective. We denote by $(X, Z)$, with $X$ in $\mathfrak{v}$ and $Z$ in $\mathfrak{z}$, the element $\exp (X+Z)$ of the group $N$.

By the Baker-Campbell-Hausdorff formula the group law is given by

$$
(X, Z)\left(X^{\prime}, Z^{\prime}\right)=\left(X+X^{\prime}, Z+Z^{\prime}+\frac{1}{2}\left[X, X^{\prime}\right]\right) \quad \forall X, X^{\prime} \in \mathfrak{v}, \quad \forall Z, Z^{\prime} \in \mathfrak{z} .
$$

The group $N$ is unimodular, and a Haar measure $d n$ on $N$ is $2^{d_{\mathfrak{z}}} d X d Z$, where $d X$ and $d Z$ are the Lebesgue measures on the real vector spaces $\mathfrak{v}$ and $\mathfrak{z}$, respectively.

Let $t$ be a positive real number. We define the homogeneous dilation $\delta_{t}$ on $N$ by the rule $\delta_{t}(X, Z)=\left(t X, t^{2} Z\right)$ for every $(X, Z)$ in $N$. It is easy to check that $\delta_{t}$ is a group automorphism and that the homogeneous dimension $Q$ of $N$ is $d_{\mathfrak{v}}+2 d_{\mathfrak{z}}$. 
As proved by Cygan [6], a homogeneous gauge on $N$ is the function $\mathcal{N}$ given by

$$
\mathcal{N}(X, Z)=\left(\frac{|X|^{4}}{16}+|Z|^{2}\right)^{1 / 4} \quad \forall(X, Z) \in N .
$$

The Iwasawa $N$-groups are characterized, among all $H$-type groups, by an algebraic condition, called the $J^{2}$-condition [4].

Definition 2.1. We say that $\mathfrak{n}$ satisfies the $J^{2}$-condition if, for any $X$ in $\mathfrak{v}$ and $Z, Z^{\prime}$ in $\mathfrak{z}$, such that $\left\langle Z, Z^{\prime}\right\rangle=0$, there exists $Z^{\prime \prime}$ in $\mathfrak{z}$ such that

$$
J_{Z} J_{Z^{\prime}} X=J_{Z^{\prime \prime}} X \text {. }
$$

For $X$ in $\mathfrak{v}$, let $\mathfrak{k}(X)$ denote the orthogonal complement of $\left(\mathbb{R} \oplus J_{\mathfrak{z}}\right) X$ in $\mathfrak{v}$. The $J^{2}$-condition is equivalent to the fact that the subspace $\mathfrak{k}(X)$ is preserved under the action of $J_{Z}$ for every $Z$ in $\mathfrak{z}$.

As we shall see in the next section, the $J^{2}$-condition is strictly linked with the geometric properties of the Cayley transform $\mathcal{C}$, mapping $N$ into the unit sphere $\mathbb{S}$ in $\mathfrak{v} \oplus \mathfrak{z} \oplus \mathbb{R}$, i.e.,

$$
\mathcal{C}: N \longrightarrow \mathbb{S}=\left\{\left(X^{\prime}, Z^{\prime}, t^{\prime}\right) \in \mathfrak{v} \oplus \mathfrak{z} \oplus \mathbb{R}:\left|X^{\prime}\right|^{2}+\left|Z^{\prime}\right|^{2}+t^{\prime 2}=1\right\} .
$$

The map $\mathcal{C}$ was introduced in [5], and it is given by

$$
\mathcal{C}(X, Z)=\frac{1}{\mathcal{B}(X, Z)}\left(\overline{\mathcal{A}}(X, Z) X, 2 Z,-1+\frac{|X|^{4}}{16}+|Z|^{2}\right) \quad \forall(X, Z) \in N,
$$

where $\mathcal{A}(X, Z)$ and $\overline{\mathcal{A}}(X, Z)$ are the linear operators on $\mathfrak{v}$ defined by

$$
\mathcal{A}(X, Z) Y=\left(1+\frac{|X|^{2}}{4}+J_{Z}\right) Y, \quad \overline{\mathcal{A}}(X, Z) Y=\left(1+\frac{|X|^{2}}{4}-J_{Z}\right) Y \quad \forall Y \in \mathfrak{v},
$$

and $\mathcal{B}(X, Z)$ is the real number

$$
\mathcal{B}(X, Z)=\left(1+\frac{|X|^{2}}{4}\right)^{2}+|Z|^{2}
$$

When the dependence on $(X, Z)$ in $N$ is clear, we shall more simply write $\mathcal{A}, \overline{\mathcal{A}}$ and $\mathcal{B}$. In the case where $N$ is an Iwasawa group, the Cayley transform is essentially the map $\bar{n} \mapsto k(\bar{n})$ defined in [9, Ch. 9, Theorem 3.8, p. 414].

Let $o=(0,0,1)$. The inverse of the Cayley transform $\mathcal{C}^{-1}: \mathbb{S} \backslash\{o\} \longrightarrow N$ is given by the rule

$$
\mathcal{C}^{-1}\left(X^{\prime}, Z^{\prime}, t^{\prime}\right)=\frac{1}{\left(1-t^{\prime}\right)^{2}+\left|Z^{\prime}\right|^{2}}\left(2\left(1-t^{\prime}+J_{Z^{\prime}}\right) X^{\prime}, 2 Z^{\prime}\right) .
$$

In the case where $N$ is an Iwasawa group, the jacobian determinant $J_{\mathcal{C}^{-1}}$ of the map $\mathcal{C}^{-1}$ has been computed in [5] and, for every $\left(X^{\prime}, Z^{\prime}, t^{\prime}\right)$ in $\mathbb{S} \backslash\{o\}$, is given by

$$
J_{\mathcal{C}^{-1}}\left(X^{\prime}, Z^{\prime}, t^{\prime}\right)=\left(\mathcal{B} \circ \mathcal{C}^{-1}\left(X^{\prime}, Z^{\prime}, t^{\prime}\right)\right)^{Q / 2}=\left(\frac{4}{\left(1-t^{\prime}\right)^{2}+\left|Z^{\prime}\right|^{2}}\right)^{Q / 2} .
$$

Clearly, in these cases, $J_{\mathcal{C}}=J_{\mathcal{C}^{-1}}^{-1} \circ \mathcal{C}=\mathcal{B}^{-Q / 2}$.

We fix orthonormal bases $\left\{E_{j}\right\}_{j=1}^{d_{\mathfrak{v}}}$ and $\left\{U_{k}\right\}_{k=1}^{d_{\mathfrak{z}}}$ of $\mathfrak{v}$ and $\mathfrak{z}$, respectively. For $X$ in $\mathfrak{v}$ and $Z$ in $\mathfrak{z}$, we write $X=\sum_{j=1}^{d_{\mathfrak{v}}} x_{j} E_{j}$ and $Z=\sum_{k=1}^{d_{\mathfrak{z}}} z_{k} U_{k}$. Given a vector $V$ in $\mathfrak{n}$, we keep the same notation for the left-invariant vector field associated to it, hence we write

$$
V f(n)=\left.\frac{d}{d t}\right|_{t=0} f(n \exp t V) \quad \forall n \in N, \quad f \in C^{\infty}(N) .
$$


It is easy to check that, for a smooth function $f$ on $N$,

$$
\begin{aligned}
& E_{j} f(X, Z)=\partial_{x_{j}} f(X, Z)+\frac{1}{2} \sum_{k=1}^{d_{3}}\left\langle J_{U_{k}} X, E_{j}\right\rangle \partial_{z_{k}} f(X, Z), \\
& U_{k} f(X, Z)=\partial_{z_{k}} f(X, Z),
\end{aligned}
$$

where $j=1, \ldots, d_{\mathfrak{v}}$ and $k=1, \ldots, d_{\mathfrak{z}}$. We write $T_{n}^{(1)}(N)$ and $T_{n}^{(2)}(N)$ for the spans of the tangent vectors at $n$ in $N$ associated respectively to the $E_{j}$ and to the $U_{k}$.

Finally, we include two explicit examples of Heisenberg type groups, namely the Heisenberg group, which satisfies the $J^{2}$-condition, and the complexified Heisenberg group, for which the $J^{2}$-condition fails.

Example 2.2. Let $\mathfrak{v}=\mathbb{C}$ and $\mathfrak{z}=\mathbb{R}$. For every $z, z_{0}$ in $\mathfrak{v}=\mathbb{C}$ and $t, t_{0}$ in $\mathfrak{z}=\mathbb{R}$, define an $H$-type algebra by setting

$$
\left[(z, t),\left(z_{0}, t_{0}\right)\right]=\operatorname{Im}\left(z_{0} \bar{z}\right), \quad\left\langle(z, t),\left(z_{0}, t_{0}\right)\right\rangle=\operatorname{Re}\left(z \overline{z_{0}}\right)+t t_{0} .
$$

Then $\mathfrak{n}=\mathfrak{v}+\mathfrak{z}$ is the 3-dimensional Heisenberg algebra. Note that $J_{t} z=i t z$ for every $z$ in $\mathfrak{v}$ and $t$ in $\mathfrak{z}$, and we can use the map $J$ to embed the sphere in $\mathbb{C}^{2}$ as follows. Denote the elements in $N$ and $\mathbb{S}$ respectively by $(z, t)$ and $\left(z^{\prime}, u^{\prime}+i t^{\prime}\right)$, where $z, z^{\prime}$ are in $\mathbb{C}, u^{\prime}, t^{\prime}$ are in $\mathbb{R}$ and $\left|z^{\prime}\right|^{2}+\left|u^{\prime}\right|^{2}+t^{\prime 2}=1$. Then the Cayley transform $\mathcal{C}$, mapping $N$ into the unit sphere $\mathbb{S}$, is given by the rule

$$
\mathcal{C}(z, t)=\left(\frac{z}{1+\frac{|z|^{2}}{4}+i t}, 1-\frac{2}{1+\frac{|z|^{2}}{4}+i t}\right) \quad \forall(z, t) \in N,
$$

and, when $\left(z^{\prime}, u^{\prime}+i t^{\prime}\right)$ is in $\mathbb{S} \backslash\{(0,1)\}$,

$$
\mathcal{C}^{-1}\left(z^{\prime}, u^{\prime}+i t^{\prime}\right)=\left(\frac{2 z^{\prime}}{1-u^{\prime}-i t^{\prime}}, \operatorname{Im}\left(-1+\frac{2}{1-u^{\prime}-i t^{\prime}}\right)\right) .
$$

Example 2.3. We define an $H$-type algebra $\mathfrak{n}=\mathfrak{v}+\mathfrak{z}$ by setting

$$
\mathfrak{v}=\mathbb{C} \times \mathbb{C}, \quad \mathfrak{z}=\mathbb{C},
$$

and, for every $X=(u, v), X_{0}=\left(u_{0}, v_{0}\right)$ in $\mathfrak{v}=\mathbb{C} \times \mathbb{C}$ and $z, z_{0}$ in $\mathfrak{z}=\mathbb{C}$,

$$
\left[X+z, X_{0}+z_{0}\right]=u v_{0}-v u_{0}, \quad\left\langle X+z, X_{0}+z_{0}\right\rangle=\operatorname{Re}\left(u \overline{u_{0}}+v \overline{v_{0}}+z \overline{z_{0}}\right) .
$$

Note that in this case the $J^{2}$-condition does not hold: indeed, $J_{z}(u, v)=(-z \bar{v}, z \bar{u})$, for every $u, v, z$ in $\mathbb{C}$. Therefore, if $z_{1}=1$ and $z_{2}=i$, it is not possible to find $z_{3}$ in $\mathfrak{z}$ such that $J_{z_{3}}=J_{z_{1}} J_{z_{2}}$. We recall 4 that if the $J^{2}$-condition holds, then $d_{\mathfrak{z}}$ must be equal to 1,3 or 7 .

Denote the elements in $N$ and $\mathbb{S}$ respectively by $(u, v, z)$ and $\left(u^{\prime}, v^{\prime}, z^{\prime}, t^{\prime}\right)$, where $u, v, z, u^{\prime}, v^{\prime}, z^{\prime}$ are in $\mathbb{C}, t^{\prime}$ is in $\mathbb{R}$ and $\left|u^{\prime}\right|^{2}+\left|v^{\prime}\right|^{2}+\left|z^{\prime}\right|^{2}+t^{\prime 2}=1$. Then the Cayley transform $\mathcal{C}$, mapping $N$ into the unit sphere $\mathbb{S}$, is given by the rule

$$
\begin{aligned}
& \mathcal{C}(u, v, z) \\
& =\mathcal{B}^{-1}\left(\left(1+\frac{|(u, v)|^{2}}{4}\right) u+z \bar{v},\left(1+\frac{|(u, v)|^{2}}{4}\right) v-z \bar{u}, 2 z, \mathcal{B}-2\left(1+\frac{|(u, v)|^{2}}{4}\right)\right),
\end{aligned}
$$

and, when $\left(u^{\prime}, v^{\prime}, z^{\prime}, t^{\prime}\right) \neq(0,0,0,1)$,

$$
\mathcal{C}^{-1}\left(u^{\prime}, v^{\prime}, z^{\prime}, t^{\prime}\right)=\frac{2}{\left(1-t^{\prime}\right)^{2}+\left|z^{\prime}\right|^{2}}\left(\left(1-t^{\prime}\right) u^{\prime}-z^{\prime} \overline{v^{\prime}},\left(1-t^{\prime}\right) v^{\prime}+z^{\prime} \overline{u^{\prime}}, z^{\prime}\right),
$$

where $|(u, v)|^{2}=|u|^{2}+|v|^{2}$ and $\mathcal{B}=\left(1+\frac{|(u, v)|^{2}}{4}\right)^{2}+|z|^{2}$. 


\section{The JaCobian of the CAYley transform}

We endow the sphere $\mathbb{S}$ with its Euclidean $S O\left(d_{\mathfrak{v}}+d_{\mathfrak{z}}+1\right)$-invariant standard metric. Note that the tangent space at the point $o$ decomposes naturally into the orthogonal sum $\mathfrak{v} \oplus \mathfrak{z}$. When $N$ is an Iwasawa group, there is a group $K$ acting orthogonally [5, Theorem 6.1] and transitively on $\mathbb{S}$. Let $M$ be the isotropy subgroup of $o$. Then the sphere may be identified with the coset $K / M$, and $\operatorname{Ad}(M)$ preserves $\mathfrak{v}$ and $\mathfrak{z}$. So the tangent space $T_{p}(\mathbb{S})$ at any point $p=k \cdot o$ decomposes orthogonally as $T_{p}^{(1)}(\mathbb{S}) \oplus T_{p}^{(2)}(\mathbb{S})$, where $T_{p}^{(1)}(\mathbb{S})=k_{*} \mathfrak{v}$ and $T_{p}^{(2)}(\mathbb{S})=k_{*} \mathfrak{z}$.

Similarly, when $N$ is a general Heisenberg type group, one can decompose the tangent space $T_{p}(\mathbb{S})$ at the point $p$ on the sphere into the orthogonal sum $T_{p}^{(1)}(\mathbb{S}) \oplus$ $T_{p}^{(2)}(\mathbb{S})$ with respect to the Euclidean metric [5], where, if $p=\left(X^{\prime}, Z^{\prime}, t^{\prime}\right)$, then $T_{p}^{(2)}(\mathbb{S}) \oplus \mathbb{R} p$ equals $\mathbb{R} X^{\prime}+J_{\mathfrak{z}} X^{\prime}$ if $Z^{\prime}=t^{\prime}=0$, and otherwise

$T_{p}^{(2)}(\mathbb{S}) \oplus \mathbb{R} p=\left\{\left(\left(u+J_{W}\right)\left(t^{\prime}-J_{Z^{\prime}}\right) X^{\prime},\left(\left|Z^{\prime}\right|^{2}+t^{\prime 2}\right) W,\left(\left|Z^{\prime}\right|^{2}+t^{\prime 2}\right) u\right): W \in \mathfrak{z}, u \in \mathbb{R}\right\}$.

In the following lemma we evaluate the differential of the Cayley transform. If $n$ is in $N, p=\mathcal{C}(n)$ and $V$ is in $\mathfrak{v}$, we use the notation

$$
\left(\mathcal{C}_{*} V\right)(p)=\left.\frac{d}{d t}\right|_{t=0} \mathcal{C}(n \exp (t V))
$$

Lemma 3.1. Let $(X, Z)$ be in $N$. If $E$ is in $\mathfrak{v}$, then $\mathcal{C}_{*} E=\left(E_{1}^{\prime}, E_{2}^{\prime}, E_{3}^{\prime}\right)$, where

$$
\begin{aligned}
& E_{1}^{\prime}(\mathcal{C}(X, Z))=\mathcal{B}^{-1}\left(\overline{\mathcal{A}} E+\frac{1}{2}\left(\langle X, E\rangle-J_{[X, E]}\right) X-\mathcal{B}^{-1}\langle\mathcal{A} X, E\rangle \overline{\mathcal{A}} X\right), \\
& E_{2}^{\prime}(\mathcal{C}(X, Z))=\mathcal{B}^{-1}\left(-2 \mathcal{B}^{-1}\langle\mathcal{A} X, E\rangle Z+[X, E]\right), \\
& E_{3}^{\prime}(\mathcal{C}(X, Z))=\mathcal{B}^{-1}\left(\frac{|X|^{2}}{4}\langle X, E\rangle+\left\langle J_{Z} X, E\right\rangle-\mathcal{B}^{-1}\langle\mathcal{A} X, E\rangle\left(-1+\frac{|X|^{4}}{16}+|Z|^{2}\right)\right) .
\end{aligned}
$$

If $U$ is in $\mathfrak{z}$, then $\mathcal{C}_{*} U=\left(U_{1}^{\prime}, U_{2}^{\prime}, U_{3}^{\prime}\right)$, where

$$
\begin{aligned}
& U_{1}^{\prime}(\mathcal{C}(X, Z))=\mathcal{B}^{-1}\left(-J_{U} X-2 \mathcal{B}^{-1}\langle Z, U\rangle \overline{\mathcal{A}} X\right), \\
& U_{2}^{\prime}(\mathcal{C}(X, Z))=2 \mathcal{B}^{-1}\left(U-2 \mathcal{B}^{-1}\langle Z, U\rangle Z\right), \\
& U_{3}^{\prime}(\mathcal{C}(X, Z))=2 \mathcal{B}^{-1}\left(\langle Z, U\rangle-2 \mathcal{B}^{-1}\langle Z, U\rangle\left(-1+\frac{|X|^{4}}{16}+|Z|^{2}\right)\right) .
\end{aligned}
$$

Proof. We write the expansion of $\mathcal{C}((X, Z)(t E, t U))$ in powers of $t$. Routine computations give the desired result.

In the following proposition we show that the $J^{2}$-condition holds if and only if the restriction of $\mathcal{C}_{*}$ to $T_{n}^{(1)}(N)$ is a multiple of an isometry.

Proposition 3.2. The following conditions are equivalent:

a) the $J^{2}$-condition holds;

b) for every $E$ in $\mathfrak{v}$ and every $p$ in $\mathbb{S} \backslash\{o\}$, the vector $\mathcal{C}_{*} E(p)$ lies in $T_{p}^{(1)}(\mathbb{S})$;

c) for every $E$ in $\mathfrak{v}$ and every $p$ in $\mathbb{S} \backslash\{o\},\left|\mathcal{C}_{*} E(p)\right|=J_{\mathcal{C}^{-1}}^{-1 / Q}(p)|E|$.

It was proved [1, 2] that if the $J^{2}$-condition holds, then $\mathcal{C}$ is a 1 -quasiconformal map; from this fact (and some extra computations) items b) and c) would follow. Here we include these elementary computations which also show the necessity of the $J^{2}$-condition.

Proof. We first prove that conditions a) and b) are equivalent. 
If $p=\left(X^{\prime}, Z^{\prime}, t^{\prime}\right)$ is a point on $\mathbb{S} \backslash\{o\}$ such that either $Z^{\prime} \neq 0$ or $t^{\prime} \neq 0$, then an orthonormal basis $\left\{Y_{i}(p)\right\}_{i=0}^{d_{\mathfrak{z}}}$ for $T_{p}^{(2)}(\mathbb{S}) \oplus \mathbb{R} p$ is given by [2]

$$
\begin{aligned}
& Y_{0}(p)=\frac{1}{\sqrt{\left|Z^{\prime}\right|^{2}+t^{\prime 2}}}\left(\left(t^{\prime}-J_{Z^{\prime}}\right) X^{\prime}, 0,\left|Z^{\prime}\right|^{2}+t^{\prime 2}\right), \\
& Y_{i}(p)=\frac{1}{\sqrt{\left|Z^{\prime}\right|^{2}+t^{\prime 2}}}\left(J_{U_{i}}\left(t^{\prime}-J_{Z^{\prime}}\right) X^{\prime},\left(\left|Z^{\prime}\right|^{2}+{t^{\prime}}^{2}\right) U_{i}, 0\right) .
\end{aligned}
$$

Let $E$ be in $\mathfrak{v}$. Then $\mathcal{C}_{*} E(p)$ is in $T_{p}^{(1)}(\mathbb{S})$ if and only if

$$
\left\langle\mathcal{C}_{*} E, Y_{i}\right\rangle=0, \quad i=0, \ldots, d_{\mathfrak{z}} .
$$

If $p=\mathcal{C}(X, Z)$ we obtain

$$
\begin{aligned}
Y_{0}(\mathcal{C}(X, Z)) & =\frac{1}{\mathcal{B}^{1 / 2} \sqrt{\mathcal{B}-|X|^{2}}}\left((\overline{\mathcal{A}} X-2 X), 0, \mathcal{B}-|X|^{2}\right), \\
Y_{i}(\mathcal{C}(X, Z)) & =\frac{1}{\mathcal{B}^{1 / 2} \sqrt{\mathcal{B}-|X|^{2}}}\left(J_{U_{i}}(\overline{\mathcal{A}} X-2 X),\left(\mathcal{B}-|X|^{2}\right) U_{i}, 0\right) .
\end{aligned}
$$

Straightforward computations using Lemma 3.1 show that $\left\langle\mathcal{C}_{*} E, Y_{0}\right\rangle=0$.

When $i=1, \ldots, d_{\mathfrak{z}}$, it is easy to compute that $\left\langle\mathcal{C}_{*} E(p), Y_{i}(p)\right\rangle$ equals

$$
\begin{aligned}
& \frac{1}{\mathcal{B}^{3 / 2} \sqrt{\mathcal{B}-|X|^{2}}}\left(\left\langle\overline{\mathcal{A}} E+\frac{1}{2}\left(\langle X, E\rangle-J_{[X, E]}\right) X-\mathcal{B}^{-1}\langle\mathcal{A} X, E\rangle \overline{\mathcal{A}} X, J_{U_{i}}(\overline{\mathcal{A}} X-2 X)\right\rangle\right. \\
& \left.\quad+\left\langle-2 \mathcal{B}^{-1}\langle\mathcal{A} X, E\rangle Z+[X, E],\left(\mathcal{B}-|X|^{2}\right) U_{i}\right\rangle\right) \\
& =\frac{1}{\mathcal{B}^{3 / 2} \sqrt{\mathcal{B}-|X|^{2}}}\left\langle[\overline{\mathcal{A}} X, \overline{\mathcal{A}} E]+\frac{1}{2}\langle X, E\rangle[\overline{\mathcal{A}} X, X]-\frac{1}{2}\left[\overline{\mathcal{A}} X, J_{[X, E]} X\right]\right. \\
& \quad-2[X, \overline{\mathcal{A}} E]+\left[X, J_{[X, E]} X\right]+2 \mathcal{B}^{-1}\langle\mathcal{A} X, E\rangle\left([X, \overline{\mathcal{A}} X]-\left(\mathcal{B}-|X|^{2}\right) Z\right) \\
& \left.+\left(\mathcal{B}-|X|^{2}\right)[X, E], U_{i}\right\rangle .
\end{aligned}
$$

Using properties (2.2) of the map $J$, one can show that

$$
\begin{aligned}
& {\left[J_{Z} X, J_{Z} E\right]=2\left\langle J_{Z} X, E\right\rangle Z-|Z|^{2}[X, E], \quad \forall X, E \in \mathfrak{v}, Z \in \mathfrak{z} .} \\
& {\left[X, J_{Z} E\right]=2\langle X, E\rangle Z+\left[J_{Z} X, E\right],}
\end{aligned}
$$

Therefore $\left\langle\mathcal{C}_{*} E(p), Y_{i}(p)\right\rangle=0$, for every $i=1, \ldots, d_{\mathfrak{z}}$, if and only if

(3.1) $\left\langle\left[J_{Z} X, J_{[X, E]} X\right]-|X|^{2}\left[J_{Z} X, E\right]-|X|^{2}\langle X, E\rangle Z, U_{i}\right\rangle=0 \quad \forall i=1, \ldots, d_{\mathfrak{z}}$.

It is enough to prove (3.1) when $|X|=1$. Let $Z=\left\langle Z, U_{i}\right\rangle U_{i}+Z^{\prime}$. By (2.2) we obtain

$$
\begin{aligned}
\left\langle\left[J_{Z} X, J_{[X, E]} X\right]\right. & \left.-\left[J_{Z} X, E\right]-\langle X, E\rangle Z, U_{i}\right\rangle \\
& =\left\langle J_{U_{i}} J_{Z} X, P_{J_{3} X} E\right\rangle-\left\langle J_{U_{i}} J_{Z} X, E\right\rangle-\langle X, E\rangle\left\langle Z, U_{i}\right\rangle \\
& =\left\langle P_{J_{\mathfrak{z}} X}\left(J_{U_{i}} J_{Z} X\right)-\left(J_{U_{i}} J_{Z}+\left\langle Z, U_{i}\right\rangle\right) X, E\right\rangle \\
& =\left\langle P_{J_{\mathfrak{z}} X}\left(J_{U_{i}} J_{Z^{\prime}} X\right)-J_{U_{i}} J_{Z^{\prime}} X, E\right\rangle .
\end{aligned}
$$

If the $J^{2}$-condition holds, the last expression vanishes and (3.1) follows. Vice versa, if for every $E$ in $\mathfrak{v}, Z=\left\langle Z, U_{i}\right\rangle U_{i}+Z^{\prime}$ in $\mathfrak{z}$, and every $i=1, \ldots, d_{\mathfrak{z}}$,

$$
\left\langle P_{J_{\mathfrak{z}} X}\left(J_{U_{i}} J_{Z^{\prime}} X\right)-J_{U_{i}} J_{Z^{\prime}} X, E\right\rangle=0,
$$

then the $J^{2}$-condition holds. 
The case where $p=\left(X^{\prime}, 0,0\right)$ is easier since $T_{p}^{(2)}(\mathbb{S}) \oplus \mathbb{R} p=\mathbb{R} X^{\prime}+J_{\mathfrak{z}} X^{\prime}$, and we omit the details.

Now we prove that conditions a) and c) are equivalent.

Let $E$ be in $\mathfrak{v}$ and $(X, Z)$ in $N$. Straightforward computations using properties (2.2) show that $\left|\mathcal{C}_{*} E(\mathcal{C}(X, Z))\right|^{2}$ equals

$$
\begin{aligned}
& \frac{1}{\mathcal{B}^{2}}(\mid \overline{\mathcal{A}} E+\frac{1}{2}\left(\langle X, E\rangle-J_{[X, E]}\right) X-\left.\mathcal{B}^{-1}\langle\mathcal{A} X, E\rangle \overline{\mathcal{A}} X\right|^{2} \\
&+\left|[X, E]-2 \mathcal{B}^{-1}\langle\mathcal{A} X, E\rangle Z\right|^{2} \\
&\left.+\left(\frac{|X|^{2}}{4}\langle X, E\rangle+\left\langle J_{Z} X, E\right\rangle-\mathcal{B}^{-1}\langle\mathcal{A} X, E\rangle\left(-1+\frac{|X|^{4}}{16}+|Z|^{2}\right)\right)^{2}\right) \\
&=\mathcal{B}^{-1}|E|^{2}-\mathcal{B}^{-2}\left(\langle X, E\rangle\left\langle J_{Z} X, E\right\rangle+\left\langle E, J_{Z} J_{[X, E]} X\right\rangle\right) .
\end{aligned}
$$

Therefore $\left|\mathcal{C}_{*} E\right|^{2} \circ \mathcal{C}=\mathcal{B}^{-1}|E|^{2}$ for every $E$ in $\mathfrak{v}$ if and only if

$$
\langle X, E\rangle\left\langle J_{Z} X, E\right\rangle+\left\langle E, J_{Z} J_{[X, E]} X\right\rangle=0 \quad \forall X, E \in \mathfrak{v}, \quad \forall Z \in \mathfrak{z} .
$$

It is enough to prove (3.2) when $E$ and $Z$ are unitary. According to the orthogonal decomposition $\mathfrak{v}=\mathbb{R} E \oplus \mathbb{R} J_{Z} E \oplus J_{\mathfrak{z} \ominus \mathbb{R} Z} E \oplus \mathfrak{k}(E)$, we write

$$
X=\langle X, E\rangle E+\left\langle X, J_{Z} E\right\rangle J_{Z} E+J_{W} E+Y .
$$

Then $[X, E]=-\left\langle X, J_{Z} E\right\rangle Z-W$ and $\left\langle E, J_{Z} J_{[X, E]} X\right\rangle+\left\langle J_{Z} X, E\right\rangle\langle E, X\rangle$ equals

$$
\begin{aligned}
& \left\langle X, J_{Z} E\right\rangle\langle E, X\rangle-\left\langle E, J_{Z} J_{W} X\right\rangle+\left\langle J_{Z} X, E\right\rangle\langle E, X\rangle \\
= & -\left\langle E, J_{Z} J_{W} X\right\rangle \\
= & -\left\langle J_{W} J_{Z} E, Y\right\rangle .
\end{aligned}
$$

The last expression vanishes for every $X$ and $E$ if and only if $J_{W} J_{Z} E$ is orthogonal to $\mathfrak{k}(E)$, for every $E$, i.e., the $J^{2}$-condition holds.

\section{Sobolev SPACES}

4.1. Sobolev spaces on $N$. Let $\Delta$ be the sublaplacian on $N$ defined by

$$
\Delta=-\sum_{j=1}^{d_{\mathfrak{v}}} E_{j}^{2},
$$

where $E_{j}$ are the left-invariant vector fields given by formula (2.3). The operator $\Delta$ is a densely defined, essentially self-adjoint, positive operator on $L^{2}(N)$. Real powers of the sublaplacian may be defined spectrally as in [3, 7].

Definition 4.1. For real $\alpha$, we define the homogeneous Sobolev space $\mathcal{H}^{\alpha}(N)$ to be the completion of $C_{c}^{\infty}(N)$, the space of smooth functions with compact support on $N$, with respect to the norm

$$
\|f\|_{\mathcal{H}^{\alpha}(N)}=\left(\Delta^{\alpha} f \mid f\right)^{1 / 2}=\left\|\Delta^{\alpha / 2} f\right\|_{2} \quad \forall f \in C_{c}^{\infty}(N) .
$$

We need the following characterization of Sobolev spaces, proved by Folland for the nonhomogeneous Sobolev spaces $\mathcal{H}^{\alpha}(N) \cap L^{2}(N)$ for $\alpha>0$; his proof can be adapted without substantial changes to the case of homogeneous Sobolev spaces of any order.

If $f$ is in $\mathcal{H}^{\alpha}(N)$, we shall write $\partial_{x_{j}} f$ and $\partial_{z_{h}} f$ for the distributional derivatives of $f$. 
Theorem 4.2 ([7, Theorem 4.10]). Let $\alpha$ be real. Then $f$ is in $\mathcal{H}^{\alpha+1}(N)$ if and only if $E_{j} f$ is in $\mathcal{H}^{\alpha}(N)$ for every $j=1, \ldots, d_{\mathfrak{v}}$; moreover the norms $\|f\|_{\mathcal{H}^{\alpha+1}(N)}$ and $\sum_{j=1}^{d_{\mathfrak{v}}}\left\|E_{j} f\right\|_{\mathcal{H}^{\alpha}(N)}$ are equivalent.

Another fundamental tool in our investigation is the following multiplier theorem, proved for the first time in [3] on some Iwasawa $N$-groups. For a real number $\gamma$ denote by $\mathcal{M}_{\gamma}(N)$ the space of functions $m$ smooth away from the identity and such that

$$
|m| \leq C \mathcal{N}^{-\gamma}, \quad\left|E_{j_{1}} E_{j_{2}} \cdots E_{j_{k}} m\right| \leq C_{k} \mathcal{N}^{-\gamma-k},
$$

where $k=1,2, \ldots$, and $1 \leq j_{1}, j_{2}, \ldots, j_{k} \leq d_{\mathfrak{v}}$.

Theorem 4.3 ([1, Theorem 3.6]). Suppose that $-Q / 2<\alpha \leq \beta<Q / 2, \gamma=\beta-\alpha$, and that $m$ is in $\mathcal{M}_{\gamma}(N)$. Then pointwise multiplication by the function $m$ defines a bounded operator from $\mathcal{H}^{\beta}(N)$ to $\mathcal{H}^{\alpha}(N)$.

4.2. Sobolev spaces on the sphere. From now on, we suppose that the $J^{2}$ condition holds, i.e., that $N$ is an Iwasawa group. As remarked previously, we shall identify $\mathbb{S}$ with the coset $K / M$.

As in 1], define the sublaplacian $\mathcal{L}$ on $\mathbb{S}$ by

$$
(\mathcal{L} f \mid f)_{\mathbb{S}}=\sum_{j=1}^{d_{\mathfrak{v}}}\left(W_{j} f \mid W_{j} f\right)_{\mathbb{S}} \quad \forall f \in C^{\infty}(\mathbb{S})
$$

where $\left\{W_{j}\right\}_{j=1}^{d_{\mathfrak{v}}}$ is an orthonormal basis for $T^{(1)}(\mathbb{S})$ defined almost everywhere on $\mathbb{S}$. The operator $\mathcal{L}$ is an essentially self-adjoint positive operator.

Via the Cayley transform, i.e., via Proposition 3.2 and polarization, it follows that for every $p$ in $\mathbb{S} \backslash\{o\}$ an orthonormal basis of $T_{p}^{(1)}(\mathbb{S})$ is obtained by normalizing the vector fields $\mathcal{C}_{*} E_{j}(p), j=1, \ldots, d_{\mathfrak{v}}$.

From now on we shall denote by $W_{j}$ the vector field

$$
W_{j}=\mathcal{C}_{*} E_{j} /\left|\mathcal{C}_{*} E_{j}\right|=J_{\mathcal{C}^{-1}}^{1 / Q} \mathcal{C}_{*} E_{j}, \quad j=1, \ldots, d_{\mathfrak{v}} .
$$

Note that $W_{j}$ are not $K$-invariant, nor are they divergence free. However an explicit formula for the sublaplacian $\mathcal{L}$ can be found.

Lemma 4.4. For every smooth function $f$ on the sphere

$$
\mathcal{L} f=-\sum_{j=0}^{d_{\mathfrak{v}}} W_{j}^{2} f+\left(\frac{1}{2}-\frac{Q}{2}\right)\left(J_{\mathcal{C}}^{-1 / Q} m_{j}\right) \circ \mathcal{C}^{-1} W_{j} f,
$$

where for $(X, Z)$ in $N$

$$
m_{j}(X, Z)=J_{\mathcal{C}}^{2 / Q}(X, Z)\left\langle\mathcal{A} X, E_{j}\right\rangle .
$$

Proof. Note that the set of functions $g$ of the form

$$
g=J_{\mathcal{C}^{-1}}^{1 / 2} G \circ \mathcal{C}^{-1}=\left(J_{\mathcal{C}}^{-1 / 2} G\right) \circ \mathcal{C}^{-1},
$$


where $G$ is a Schwartz function on $N$, is dense in $L^{2}(\mathbb{S})$. For such functions $g$ integrating by parts the boundary term vanishes and therefore

$$
\begin{aligned}
(\mathcal{L} f \mid g)_{\mathbb{S}} & =\sum_{j=1}^{d_{\mathfrak{v}}}\left(W_{j} f \mid W_{j} g\right)_{\mathbb{S}} \\
& =\sum_{j=1}^{d_{\mathfrak{v}}}\left(J_{\mathcal{C}^{-1}}^{1 / Q} \mathcal{C}_{*} E_{j} f \mid J_{\mathcal{C}^{-1}}^{1 / Q} \mathcal{C}_{*} E_{j} g\right)_{\mathbb{S}} \\
& =\sum_{j=1}^{d_{\mathfrak{v}}}\left(J_{\mathcal{C}}^{1-2 / Q} E_{j}(f \circ \mathcal{C}) \mid E_{j}(g \circ \mathcal{C})\right)_{N} \\
& =-\sum_{j=1}^{d_{\mathfrak{v}}}\left(E_{j}\left(J_{\mathcal{C}}^{1-1 / Q} J_{\mathcal{C}}^{-1 / Q} E_{j}(f \circ \mathcal{C})\right) \mid g \circ \mathcal{C}\right)_{N} \\
& =-\sum_{j=1}^{d_{\mathfrak{v}}}\left(\left(W_{j}^{2} f\right) \circ \mathcal{C}+\left(\frac{1}{2}-\frac{Q}{2}\right) J_{\mathcal{C}}^{1 / Q}\left\langle\mathcal{A} X, E_{j}\right\rangle\left(W_{j} f\right) \circ \mathcal{C} \mid g \circ \mathcal{C} J_{\mathcal{C}}\right)_{N} \\
& =\left(-\sum_{j=1}^{d_{\mathfrak{v}}} W_{j}^{2} f+\left(\frac{1}{2}-\frac{Q}{2}\right)\left(J_{\mathcal{C}}^{-1 / Q} m_{j}\right) \circ \mathcal{C}^{-1} W_{j} f \mid g\right)_{\mathbb{S}}
\end{aligned}
$$

as required.

Remark. In the real case, i.e., when $\mathfrak{v}=0$ or $\mathfrak{z}=0$, the operator $\mathcal{L}$ is the LaplaceBeltrami operator on the real Euclidean sphere. In the complex case, i.e., when $N$ is the Heisenberg group, the operator $\mathcal{L}$ is the laplacian considered by Geller in 8 on the complex sphere.

The sublaplacians $\Delta$ and $\mathcal{L}$ are linked by the equality

$$
J_{\mathcal{C}}^{-1 / 2-1 / Q} \Delta\left(J_{\mathcal{C}}^{1 / 2-1 / Q} f \circ \mathcal{C}\right)=((\mathcal{L}+b) f) \circ \mathcal{C} \quad \forall f \in C^{\infty}(\mathbb{S}),
$$

where $b=(Q-2) d_{\mathfrak{v}} / 4$ and real powers of the conformal sublaplacian $\mathcal{L}+b$ may be defined spectrally (see [1]).

Definition 4.5. For real $\alpha$, we define the Sobolev space $\mathcal{H}^{\alpha}(\mathbb{S})$ as the completion of the space of smooth functions on $\mathbb{S}$, with respect to the norm

$$
\|f\|_{\mathcal{H}^{\alpha}(\mathbb{S})}=\left((\mathcal{L}+b)^{\alpha} f \mid f\right)_{\mathbb{S}}^{1 / 2}=\left\|(\mathcal{L}+b)^{\alpha / 2} f\right\|_{L^{2}(\mathbb{S})} \quad \forall f \in C^{\infty}(\mathbb{S}) .
$$

If $\alpha \geq 2$, then $f$ is in $\mathcal{H}^{\alpha}(\mathbb{S})$ if and only if $f$ is in $L^{2}(\mathbb{S})$ and $\mathcal{L} f$ is in $\mathcal{H}^{\alpha-2}(\mathbb{S})$; moreover the norms $\|f\|_{\mathcal{H}^{\alpha}(\mathbb{S})}$ and $\|f\|_{L^{2}(\mathbb{S})}+\|\mathcal{L} f\|_{\mathcal{H}^{\alpha-2}(\mathbb{S})}$ are equivalent.

An important property of these Sobolev spaces is the following.

Theorem 4.6 (1]). Let $-Q / 2<\alpha<Q / 2$. The map

$$
T_{\alpha}: f \longmapsto J_{\mathcal{C}}^{1 / 2-\alpha / Q} f \circ \mathcal{C}
$$

is a bounded invertible operator from $\mathcal{H}^{\alpha}(\mathbb{S})$ to $\mathcal{H}^{\alpha}(N)$.

Via the previous result, we can prove an analogue of Theorem 4.2. We remark that the techniques employed by Folland in the proof of Theorem 4.2 cannot be used in our case since the vector fields $W_{j}$ are not $K$-invariant. 
Proposition 4.7. Let $\alpha \in[1, Q / 2)$. Then $f$ is in $\mathcal{H}^{\alpha}(\mathbb{S})$ if and only if $f$ is in $L^{2}(\mathbb{S})$ and $W_{j} f$ is in $\mathcal{H}^{\alpha-1}(\mathbb{S})$ for every $j=1, \ldots, d_{\mathfrak{v}} ;$ moreover the norm $\|f\|_{\mathcal{H}^{\alpha}(\mathbb{S})}$ is equivalent to $\|f\|_{L^{2}(\mathbb{S})}+\sum_{j=1}^{d_{\mathfrak{v}}}\left\|W_{j} f\right\|_{\mathcal{H}^{\alpha-1}(\mathbb{S})}$.

Proof. Suppose that $f$ is in $\mathcal{H}^{\alpha}(\mathbb{S})$. Then $f$ is in $L^{2}(\mathbb{S})$ and, by Theorem 4.6, we only need to check that $T_{\alpha-1}\left(W_{j} f\right)$ is in $\mathcal{H}^{\alpha-1}(N)$. We have

$$
\begin{aligned}
T_{\alpha-1}\left(W_{j} f\right) & =J_{\mathcal{C}}^{1 / 2-(\alpha-1) / Q}\left(W_{j} f\right) \circ \mathcal{C} \\
& =J_{\mathcal{C}}^{1 / 2-\alpha / Q} E_{j}(f \circ \mathcal{C}) \\
& =E_{j}\left(J_{\mathcal{C}}^{1 / 2-\alpha / Q} f \circ \mathcal{C}\right)-E_{j}\left(J_{\mathcal{C}}^{1 / 2-\alpha / Q}\right) f \circ \mathcal{C} \\
& =E_{j}\left(T_{\alpha} f\right)+\left(\frac{Q}{4}-\frac{\alpha}{2}\right) J_{\mathcal{C}}^{2 / Q}\left\langle\mathcal{A} X, E_{j}\right\rangle T_{\alpha} f \\
& =E_{j}\left(T_{\alpha} f\right)+\left(\frac{Q}{4}-\frac{\alpha}{2}\right) m_{j} T_{\alpha} f .
\end{aligned}
$$

It is easy to check that the function $m_{j}$ is smooth and bounded by a constant multiple of $\mathcal{N}^{-1}$; moreover for every $n=1,2, \ldots$,

$$
\left|E_{s_{1}} E_{s_{2}} \cdots E_{s_{n}} m_{j}\right| \leq C_{n} \mathcal{N}^{-1-n}, \quad \text { where } \quad s_{1}, s_{2}, \ldots, s_{n} \in\left\{1,2, \ldots, d_{\mathfrak{v}}\right\} .
$$

Therefore, by the multiplier Theorem 4.3 and Theorem 4.6 we obtain that when $-\frac{Q}{2}+1<\alpha<\frac{Q}{2}$

$$
\begin{aligned}
\left\|W_{j} f\right\|_{\mathcal{H}^{\alpha-1}(\mathbb{S})} & \leq C\left\|T_{\alpha-1}\left(W_{j} f\right)\right\|_{\mathcal{H}^{\alpha-1}(N)} \\
& \leq C\left(\left\|E_{j}\left(T_{\alpha} f\right)\right\|_{\mathcal{H}^{\alpha-1}(N)}+\left\|m_{j} T_{\alpha} f\right\|_{\mathcal{H}^{\alpha-1}(N)}\right) \\
& \leq C\left\|T_{\alpha} f\right\|_{\mathcal{H}^{\alpha}(N)} \\
& \leq C\|f\|_{\mathcal{H}^{\alpha}(\mathbb{S})} .
\end{aligned}
$$

Hence $W_{j} f$ is in $\mathcal{H}^{\alpha-1}(\mathbb{S})$ and $\|f\|_{L^{2}(\mathbb{S})}+\sum_{j=1}^{d_{\mathfrak{v}}}\left\|W_{j} f\right\|_{\mathcal{H}^{\alpha-1}(\mathbb{S})} \leq C\|f\|_{\mathcal{H}^{\alpha}(\mathbb{S})}$.

Vice versa, suppose that $\alpha \geq 1, f$ is in $L^{2}(\mathbb{S})$ and $W_{j} f$ is in $\mathcal{H}^{\alpha-1}(\mathbb{S})$ when $j=1, \ldots, d_{\mathfrak{v}}$. We need to show that $\mathcal{L} f$ is in $\mathcal{H}^{\alpha-2}(\mathbb{S})$. By what we have just proved, $W_{j}^{2} f$ is in $\mathcal{H}^{\alpha-2}(\mathbb{S})$ and $\left\|W_{j}^{2} f\right\|_{\mathcal{H}^{\alpha-2}(\mathbb{S})} \leq C\left\|W_{j} f\right\|_{\mathcal{H}^{\alpha-1}(\mathbb{S})}$.

Using the explicit formula of the sublaplacian $\mathcal{L}$ proved in Lemma 4.4, it is enough to show that the function $\left(J_{\mathcal{C}}^{-1 / Q} m_{j}\right) \circ \mathcal{C}^{-1} W_{j} f$ is in $\mathcal{H}^{\alpha-2}(\mathbb{S})$ when $j=$ $1, \ldots, d_{\mathfrak{v}}$.

We first use Theorem 4.6. second we note as before that the function $m_{j}$ multiplies $\mathcal{H}^{\alpha-1}(N)$ into $\mathcal{H}^{\alpha-2}(N)$ by Theorem 4.3, finally we use Theorem 4.6 again. In formulae:

$$
\begin{aligned}
\left\|\left(J_{\mathcal{C}}^{-1 / Q} m_{j}\right) \circ \mathcal{C}^{-1} W_{j} f\right\|_{\mathcal{H}^{\alpha-2}(\mathbb{S})} & \leq C\left\|T_{\alpha-2}\left(\left(J_{\mathcal{C}}^{-1 / Q} m_{j}\right) \circ \mathcal{C}^{-1} W_{j} f\right)\right\|_{\mathcal{H}^{\alpha-2}(N)} \\
& =C\left\|m_{j} T_{\alpha-1}\left(W_{j} f\right)\right\|_{\mathcal{H}^{\alpha-2}(N)} \\
& \leq C\left\|T_{\alpha-1}\left(W_{j} f\right)\right\|_{\mathcal{H}^{\alpha-1}(N)} \\
& \leq C\left\|W_{j} f\right\|_{\mathcal{H}^{\alpha-1}(\mathbb{S})} .
\end{aligned}
$$


Hence $\mathcal{L} f$ is in $\mathcal{H}^{\alpha-2}(\mathbb{S})$ and

$$
\begin{aligned}
\|f\|_{\mathcal{H}^{\alpha}(\mathbb{S})} & \leq C\left(\|\mathcal{L} f\|_{\mathcal{H}^{\alpha-2}(\mathbb{S})}+\|f\|_{L^{2}(\mathbb{S})}\right) \\
& \leq C\left(\sum_{j=1}^{d_{\mathfrak{v}}}\left\|W_{j} f\right\|_{\mathcal{H}^{\alpha-1}(\mathbb{S})}+\|f\|_{L^{2}(\mathbb{S})}\right),
\end{aligned}
$$

as required.

\section{REFERENCES}

1. F. Astengo, M. Cowling, and B. Di Blasio, The Cayley transform and uniformly bounded representations, J. Funct. Anal. 213 (2004), 241-269. MR2078626 (2005e:22013)

2. A. D. Banner, Some properties of boundaries of symmetric spaces of rank one, Geom. Dedicata 88 (2001), 113-133. MR1877212 (2003a:22008)

3. M. Cowling, Unitary and uniformly bounded representations of some simple Lie groups, in: Harmonic analysis and group representations, C.I.M.E., 49-128, Liguori, Napoli, 1982. MR.0777340 (86h:22012)

4. M. Cowling, A. H. Dooley, A. Korányi, and F. Ricci, H-type groups and Iwasawa decompositions, Adv. Math. 87 (1991), 1-41. MR.1102963 (92e:22017)

5. M. Cowling, A. H. Dooley, A. Korányi, and F. Ricci, An approach to symmetric spaces of rank one via groups of Heisenberg type, J. Geom. Anal. 8 (1998), 199-237. MR.1705176 (2000m:53071)

6. J. Cygan, Subadditivity of homogeneous norms on certain nilpotent Lie groups, Proc. Amer. Math. Soc. 83 (1981), 69-70. MR0619983 (82k:22009)

7. G. B. Folland, Subelliptic estimates and function spaces on nilpotent Lie groups, Ark. Mat. 13 (1975), 161-207. MR0494315 (58:13215)

8. D. Geller, The Laplacian and the Kohn Laplacian for the sphere, J. Differential Geom. 15 (1980), 417-435. MR0620896 (82i:35132)

9. S. Helgason, Differential Geometry, Lie Groups, and Symmetric Spaces, Pure and Applied Mathematics, 80. Academic Press, Inc., New York, London, 1978. MR0514561 (80k:53081)

10. A. Kaplan, Fundamental solution for a class of hypoelliptic PDE generated by composition of quadratic forms, Trans. Amer. Math. Soc. 258 (1980), 147-153. MR0554324 (81c:58059)

11. A. Korányi and H. M. Reimann, Quasiconformal mappings on the Heisenberg group, Invent. Math. 80 (1985), 309-338. MR0788413 (86m:32035)

12. G. D. Mostow Strong rigidity of locally symmetric spaces, Annals of Mathematics Studies 78, Princeton University Press, Princeton, N.J., 1973. MR0385004(52:5874)

13. P. Pansu, Métriques de Carnot-Carathéodory et quasiisométries des espaces symétriques de rang un, Ann. of Math. 129 (1989), 1-60. MR0979599 (90e:53058)

Dipartimento di Matematica, Università di Genova, 16146 Genova, Italia

E-mail address: astengo@dima.unige.it

Dipartimento di Matematica, Università di Roma "Tor Vergata", 00133 Roma, Italia

E-mail address: diblasio@mat.uniroma2.it 